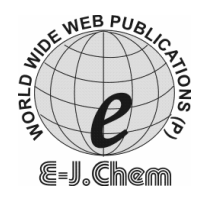

http://www.e-journals.net
ISSN: 0973-4945; CODEN ECJHAO

E-Journal of Chemistry 2009, 6(3), 759-762

\title{
Design, Synthesis and Evaluation of Schiff's Bases of 4-Chloro-3-coumarin aldehyde as Antimicrobial Agents
}

\author{
SHRIRAM BAIRAGI, ASHOK BHOSALE and MEENAKSHI N DEODHAR* \\ Department of Pharmaceutical Chemistry, \\ SGRS College of Pharmacy, Saswad, Pune - 412301, India. \\ meenakshideodhar@yahoo.com
}

Received 7 November 2008; Accepted 12 December 2008

\begin{abstract}
Chloro-2-oxo-2H-chromene-3-carbaldehyde (2) was reacted with different anilines in rectified spirit as solvent to yield a series of the title compounds i.e. 4-chloro-3-((substituted-phenylimino) methyl)-2H-chromen-2one (3a-i). These compounds were charaterised on the basis of their spectral (IR, ${ }^{1} \mathrm{H}$ NMR) data and evaluated for antimicrobial activity in vitro against gram positive and gram negative bacteria and fungi. Compound $\mathbf{3 C}$ was found to be most active with an MIC of $15 \mu \mathrm{g} / \mathrm{mL}$ against all the tested organisms.
\end{abstract}

Keywords: Coumarins, Schiff's base, Antibacterial, Antifungal activity.

\section{Introduction}

There are a number of reports that natural and synthetic coumarin derivatives posses antimicrobial activity $^{1-2}$. Novobiocin and chlorobiocin are established antimicrobials containing a coumarin skeleton. A number of the amino substituted coumarin derivatives have been reported as the inhibitors of DNA Gyrase as potential antibacterials ${ }^{3}$. Elgamal et $a l^{4}$ have reported synthesis and antimicrobial properties of hydrazino derivatives of 5-chloro-6-formylfuranocoumarins. In the present work, we report the synthesis of schiff's bases of 4-chloro-3coumarinaldehyde and their antimicrobial activity against fungi, gram positive and gram negative bacteria.

\section{Experimental}

All the chemicals used in the synthesis were of laboratory grade. The melting points were determined in open capillary on Veego (Model: VMP-D) electronic apparatus and are uncorrected. The IR spectra of synthesized compounds were recorded on Shimadzu 8400-S FT-IR spectrophotometer using potassium bromide. The ${ }^{1} \mathrm{H}$ NMR was recorded in $\mathrm{CDCl}_{3}$ using NMR Varian-Mercury $300 \mathrm{MHz}$ spectrometer and chemical shifts are given in parts per millions, downfield from tetramethylsilane (TMS) as an internal standard. To monitor the reactions, as well as, to establish the identity and purity of reactants and products, 
thin layer chromatography was performed on microscopic glass slides $(2 \times 7.5 \mathrm{~cm})$ coated with silica gel-G, using toluene-methanol and chloroform-ethyl acetate, as the solvent systems and the spots were visualized by exposure to iodine vapours.

\section{Synthesis of 4-chloro-3-((substituted phenylimino) methyl)-2H-chromen-2-one (3a-i)}

\section{General procedure}

In $100 \mathrm{~mL}$ flask fitted with a reflux condenser, $5.0 \mathrm{mmol}$ of 4-chloro-3-coumarinaldehyde (2), $5.0 \mathrm{mmol}$ of aniline and $20 \mathrm{~mL}$ of rectified spirit were taken. The solution was refluxed for 20 min. Water was then added to the hot solution until cloudiness persisted and then kept aside to cool. The separated oil was induced to crystallize by rubbing with glass rod. The solid was collected by filtration, washed well with water, dried and recrystallised from aqueous methanol. The physical and spectral data is provided in Table $1 \& 2$ respectively.

Table 1. Physical data of Schiff's bases.

\begin{tabular}{cccccc}
\hline $\begin{array}{c}\text { Compd. } \\
\text { No. }\end{array}$ & $\mathrm{R}$ & $\begin{array}{c}\text { Molecular } \\
\text { weight }\end{array}$ & $\begin{array}{c}\text { Melting point, } \\
{ }^{\circ} \mathrm{C}\end{array}$ & $\begin{array}{c}\mathrm{R}_{\mathrm{f}} \text { Value } \\
\left(\mathrm{CHCl}_{3}: \mathrm{MeOH}, 9: 1\right)\end{array}$ & $\begin{array}{c}\text { Percentage } \\
\text { yield, \% }\end{array}$ \\
\hline 3a & $2-\mathrm{NO}_{2}$ & 328.71 & $295-296$ & 0.52 & 76.68 \\
3b & $4-\mathrm{NO}_{2}$ & 328.71 & $162-164$ & 0.63 & 67.48 \\
3c & $4-\mathrm{Cl}$ & 318.15 & $167-168$ & 0.62 & 75.94 \\
3d & $3-\mathrm{OCH}_{3}$ & 313.74 & $167-168$ & 0.65 & 73.71 \\
3e & $4-\mathrm{OCH}_{3}$ & 313.74 & $126-127$ & 0.58 & 60.89 \\
3f & $2-\mathrm{OCH}_{3}$ & 313.74 & $119-121$ & 0.64 & 70.94 \\
3g & $3-\mathrm{CH}_{3}$ & 297.74 & $165-170$ & 0.58 & 64.18 \\
3h & $4-\mathrm{CH}_{3}$ & 297.74 & $203-205$ & 0.61 & 67.56 \\
3i & $\mathrm{H}$ & 283.71 & $179-180$ & 0.68 & 78.01 \\
\hline
\end{tabular}

Table 2. Spectral data of Schiff's bases.

\begin{tabular}{|c|c|c|c|}
\hline $\begin{array}{l}\text { Code } \\
\text { No. }\end{array}$ & $\mathrm{R}$ & $\mathrm{IR}(\mathrm{KBr}), \mathrm{cm}^{-1}$ & $\begin{array}{c}{ }^{1} \mathrm{H} \mathrm{NMR}\left(\mathrm{CDCl}_{3}\right) \\
\delta \mathrm{ppm}\end{array}$ \\
\hline & & \multicolumn{2}{|c|}{ 3078.4(CH),1739.8(CO), 1602.9(C=N). $8.75(\mathrm{~s}, 1 \mathrm{H},-\mathrm{CH}=\mathrm{N}-)$} \\
\hline $\mathbf{3 a}$ & 2 & $1180.4(\mathrm{C}-\mathrm{N}), 796.1(\mathrm{C}-\mathrm{Cl})$ & 7.82-7.99(d, 8H,Ar-H), \\
\hline $3 \mathbf{b}$ & $4-\mathrm{NO}_{2}$ & $\begin{array}{l}3082.3,3051.4(\mathrm{CH}), 1716.7 \\
(\mathrm{CO}), 1519.9(\mathrm{C}=\mathrm{N}), 1340.5 \\
(\mathrm{C}-\mathrm{N}), 758.0(\mathrm{C}-\mathrm{Cl})\end{array}$ & $\begin{array}{l}8.75(\mathrm{~s}, 1 \mathrm{H},-\mathrm{CH}=\mathrm{N}-) \\
7.60-7.45(\mathrm{~m}, 8 \mathrm{H}, \mathrm{Ar}-\mathrm{H})\end{array}$ \\
\hline 3c & $4-\mathrm{Cl}$ & $\begin{array}{l}3117,3080,3063,(\mathrm{CH}), 1693(\mathrm{CO}) \\
1541.1(\mathrm{C}=\mathrm{N}), 717.5(\mathrm{C}-\mathrm{Cl})\end{array}$ & $\begin{array}{l}8.75(\mathrm{~s}, 1 \mathrm{H},-\mathrm{CH}=\mathrm{N}-) \\
7.60-7.57(\mathrm{~m}, 8 \mathrm{H}, \mathrm{Ar}-\mathrm{H})\end{array}$ \\
\hline 3d & $3-\mathrm{OCH}_{3}$ & $\begin{array}{l}\text { 3066.9, 3016.7 }(\mathrm{CH}), 1739(\mathrm{CO}) \\
1473.6(\mathrm{C}=\mathrm{N}), 759.9(\mathrm{C}-\mathrm{Cl})\end{array}$ & $\begin{array}{l}8.75(\mathrm{~s}, 1 \mathrm{H},-\mathrm{CH}=\mathrm{N}-), 7.82-7.45 \\
(\mathrm{~m}, 8 \mathrm{H}, \mathrm{Ar}-\mathrm{H}), 3.43\left(\mathrm{~s}, 3 \mathrm{H}, \mathrm{OCH}_{3}\right) .\end{array}$ \\
\hline $3 \mathbf{e}$ & $4-\mathrm{OCH}_{3}$ & $\begin{array}{l}24(\mathrm{CH}), 1714(\mathrm{CO}) \\
\mathrm{C}=\mathrm{N}), 754.1(\mathrm{C}-\mathrm{Cl})\end{array}$ & $\begin{array}{l}8.75(\mathrm{~s}, 1 \mathrm{H},-\mathrm{CH}=\mathrm{N}-), 8.19-7.57(\mathrm{~m} \\
8 \mathrm{H}, \mathrm{Ar}-\mathrm{H}), 3.43\left(\mathrm{~s}, 3 \mathrm{H},-\mathrm{OCH}_{3}\right)\end{array}$ \\
\hline 3f & $2-\mathrm{OCH}_{3}$ & $\begin{array}{l}\text { 3061.1(CH), 1739.8(CO), } \\
\text { 1602.5(C=N), 769.9(C-Cl). }\end{array}$ & $\begin{array}{l}8.75(\mathrm{~s}, 1 \mathrm{H},-\mathrm{CH}=\mathrm{N}-), 8.19-7.57(\mathrm{~m}, \\
8 \mathrm{H}, \mathrm{Ar}-\mathrm{H}), 3.43\left(\mathrm{~s}, 3 \mathrm{H},-\mathrm{OCH}_{3}\right)\end{array}$ \\
\hline $3 g$ & $3-\mathrm{CH}_{3}$ & $\begin{array}{l}3340.8,3061.1(\mathrm{CH}) \\
1730.9(\mathrm{CO}), 1504.5(\mathrm{C}=\mathrm{N}), 754.1(\mathrm{C}-\mathrm{Cl})\end{array}$ & $\begin{array}{l}8.75(\mathrm{~s}, 1 \mathrm{H},-\mathrm{CH}=\mathrm{N}-), 7.60-7.57(\mathrm{~m}, 8 \mathrm{H}, \\
. \mathrm{Ar}-\mathrm{H}), 2.44\left(\mathrm{~s}, 3 \mathrm{H}, \mathrm{CH}_{3}\right) .\end{array}$ \\
\hline $3 h$ & $4-\mathrm{CH}_{3}$ & $\begin{array}{l}3117.0,3063(\mathrm{CH}), 1693.5(\mathrm{CO}) \\
1541.1(\mathrm{C}=\mathrm{N}), 717.5(\mathrm{C}-\mathrm{Cl})\end{array}$ & $\begin{array}{l}8.75(\mathrm{~s}, 1 \mathrm{H},-\mathrm{CH}=\mathrm{N}-), 7.60-7.57(\mathrm{~m}, 8 \mathrm{H}, \\
\mathrm{Ar}-\mathrm{H}), 2.44\left(\mathrm{~s}, 3 \mathrm{H}, \mathrm{CH}_{3}\right) .\end{array}$ \\
\hline $3 \mathbf{i}$ & $\mathrm{H}$ & $\begin{array}{l}\text { 3061.1, 2677.2(CH), 1741(CO), } \\
1500.6(\mathrm{C}=\mathrm{N}), 780.3(\mathrm{C}-\mathrm{Cl})\end{array}$ & $\begin{array}{l}8.75(\mathrm{~s}, 1 \mathrm{H},-\mathrm{CH}=\mathrm{N}-) \\
8.19-7.57(\mathrm{~m}, 8 \mathrm{H}, \mathrm{Ar}-\mathrm{H}) .\end{array}$ \\
\hline
\end{tabular}




\section{Antimicrobial activity}

\section{Zone of inhibition}

All the synthesized compounds were tested for their antimicrobial activity by the agar cup plate method. The organisms used were gram positive bacteria Staphylococcus aureus (ATCC 29737) and B. subtilis (ATCC 2063), gram negative bacteria E. coli (ATCC 20931) and the fungi Aspergillus niger (ATCC 16404) and Candida albicans (ATCC 10231). Amoxicillin was standard for antibacterial activity and fluconazole for antifungal activity. The results are given in Table 3.

Table 3. Antimicrobial activity of Schiff's bases.

\begin{tabular}{ccccccc}
\hline \multirow{2}{*}{ S. No. Compound } & \multicolumn{5}{c}{$\begin{array}{c}\text { Zone of inhibition in } \mathrm{mm}, 50 \mu \mathrm{g} / 10 \mu \mathrm{L} \\
\text { Bacteria }\end{array}$} \\
\cline { 3 - 7 } & & E.Coli & B.Subtilis & S.Aureus & A.Niger & C.Albicans \\
\hline 1. & 3a & 25 & 22 & 24 & 19 & 15 \\
2. & 3b & 24 & 23 & 21 & 17 & 16 \\
3. & 3c & 32 & 28 & 30 & 22 & 21 \\
4. & 3d & 21 & 23 & 20 & 15 & 14 \\
5. & 3e & 17 & 21 & 19 & 14 & 20 \\
6. & 3f & 18 & 20 & 17 & 15 & 19 \\
7. & 3g & 22 & 24 & 26 & 18 & 17 \\
8. & 3h & 21 & 22 & 24 & 19 & 16 \\
9. & 3i & 18 & 14 & 17 & 13 & 11 \\
10. & Amoxycillin & 35 & 30 & 32 & - & - \\
11. & Fluconazole & - & - & - & 24 & 22 \\
\hline
\end{tabular}

\section{Minimum inhibitory concentration}

The Minimum inhibitory concentration (MIC) against the above organisms was determined by the method of serial dilutions. The results are given in Table 4 .

Table 4. Antimicrobial activity (MIC) of Schiff's bases.

\begin{tabular}{ccccccc}
\hline S. & \multirow{2}{*}{ Compound } & \multicolumn{3}{c}{ Bacteria } & \multicolumn{2}{c}{ Fungi } \\
No. & & E.Coli & B. Subtilis & S. Aureus & A.Niger & C. Albicans \\
\hline 1. & 3a & 20 & 20 & 20 & 35 & 35 \\
2. & 3b & 20 & 20 & 20 & 30 & 35 \\
3. & $\mathbf{3 c}$ & 15 & 15 & 15 & 15 & 15 \\
4. & $\mathbf{3 d}$ & 20 & 20 & 20 & 30 & 30 \\
5. & $\mathbf{3 e}$ & 25 & 25 & 25 & 30 & 25 \\
6. & $\mathbf{3 f}$ & 25 & 25 & 25 & 30 & 30 \\
7. & $\mathbf{3 g}$ & 20 & 25 & 25 & 30 & 30 \\
8. & $\mathbf{3 h}$ & 30 & 35 & 20 & 30 & 30 \\
9. & $\mathbf{3 i}$ & 30 & 25 & 25 & 30 & 30 \\
\hline
\end{tabular}

\section{Results and Discussion}

4-Chloro-3-coumarinaldehyde (2) was prepared by Vilsmeier- Haack reaction from 4hydroxycoumarin. This in turn was synthesized from $o$-hydroxyacetophenone, diethylcarbonate and sodium using reported procedure. The schiff's bases, 4-chloro-3((substituted-phenylimino)methyl-2H-chromen-2-one (3a-i) were synthesized by reacting 2 
with substituted anilines in rectified spirit. These were obtained as solids melting in the range $126-296{ }^{0} \mathrm{C}$. The solid state $\mathrm{IR}\left(\mathrm{KBr}, \mathrm{cm}^{-1}\right)$ spectra of these compounds reveal a characteristic aromatic stretch between $2900-3100 \mathrm{~cm}^{-1}$. Sharp carbonyl C=O stretching vibrations for the lactone carbonyl are seen around $1620-1741 \mathrm{~cm}^{-1}$. The stretching vibrations for $\mathrm{C}=\mathrm{N}$ group are present at around $1473-1602 \mathrm{~cm}^{-1}$. The ${ }^{1} \mathrm{H} \mathrm{NMR}\left(\mathrm{CDCl}_{3}, \mathrm{ppm}\right)$ data of all compounds reveal peak between 7-8.75 for the aromatic protons. Presence of characteristic singlet at 8.75 assigned to the proton attached to the imine forming carbon and absence of peak by aldehydic proton confirms formation of schiff's base.<smiles>CCOC(=O)OC(C)C</smiles>
o-Hydroxyacetophenone

Diethylcarbonate<smiles>O=c1cc(O)c2ccccc2o1</smiles>

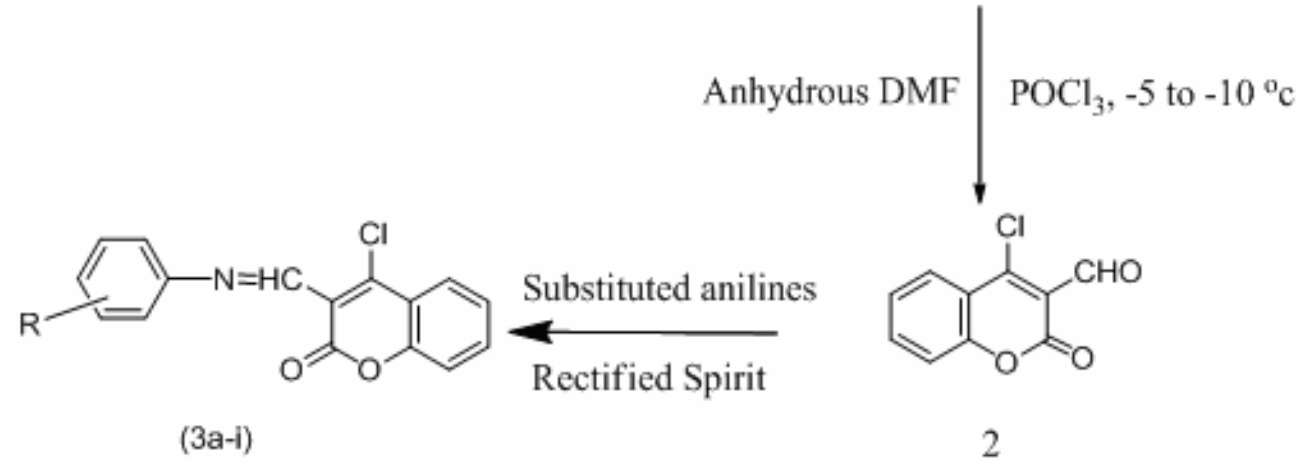

\section{Scheme 1}

All compounds have shown moderate activity as compared to standard. In general, the order of antibacterial activity of the substituents at the $4^{\text {th }}$ position of phenyl ring was observed as $\mathrm{Cl}>\mathrm{NO}_{2}>\mathrm{CH}_{3}>\mathrm{OCH}_{3}$. Compound 3c exhibited highest zone of inhibition and least MIC $(15 \mu \mathrm{g} \mathrm{m} / \mathrm{mL})$ against all organisms.

\section{Conclusion}

From the antimicrobial data of the synthesised compounds, we can conclude that the Schiff's bases of 4-chloro-3-coumarinaldehyde hold promise as antimicrobial agents after further development.

\section{References}

1. Zalfiqar A and Nasim H, Indian J Chem., 2007, 46B, 1322.

2. Bernadette S, Denis A and Kevin K, Inorg Chim Acta., 2006, 359, 3976.

3. Laurin P, Ferroud D, Klich M, Dupuis-Haelin C, Mauvais P, Lassaigne P, Bonnefoy A and Musicki B, Bioorg Med Chem Lett., 1999, 9, 2079.

4. Abou-Melha K and Faruk H, J Iran Chem Soc., 2008, 5, 1,122.

5. Braccio M, Grossi G, Roma G Signorello M and Leancini G, Eur J Med Chem., 2004, 39, 337.

6. Khan I and Kulkarni M, Eur J Med Chem., 2005, 40, 1168. 


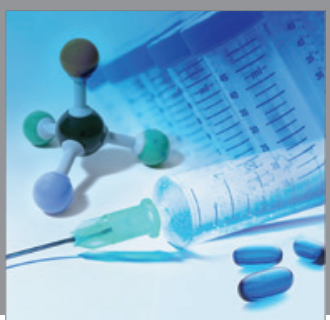

International Journal of

Medicinal Chemistry

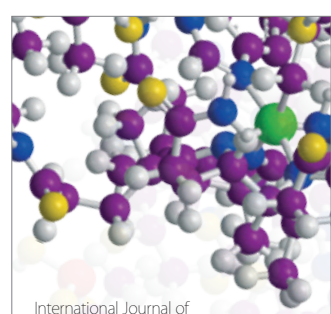

Carbohydrate Chemistry

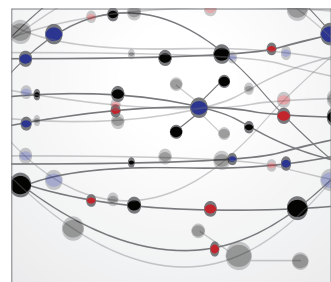

The Scientific World Journal
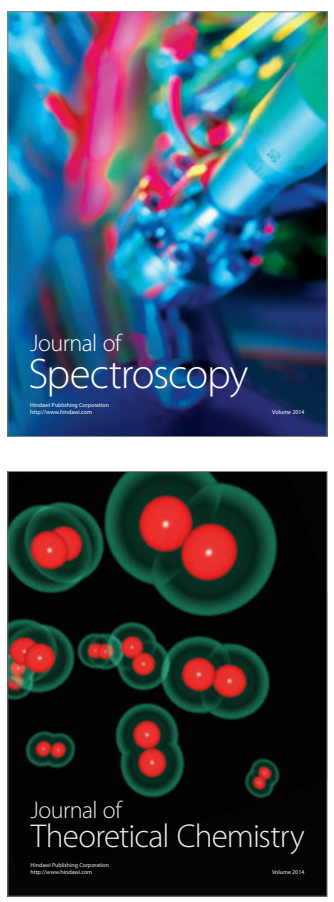
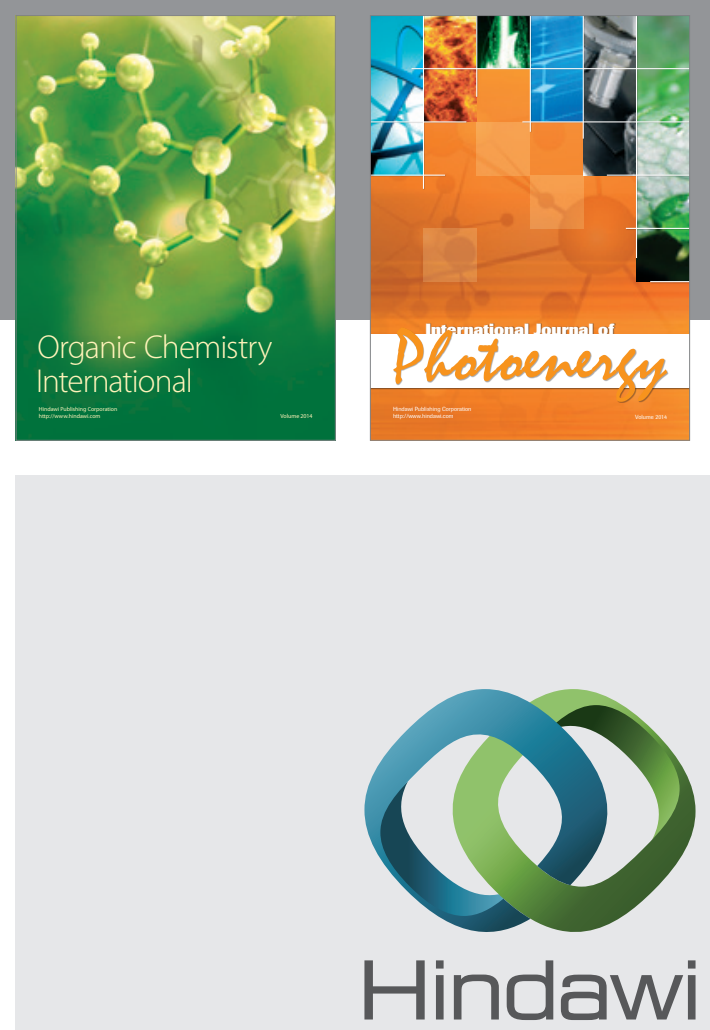

Submit your manuscripts at

http://www.hindawi.com
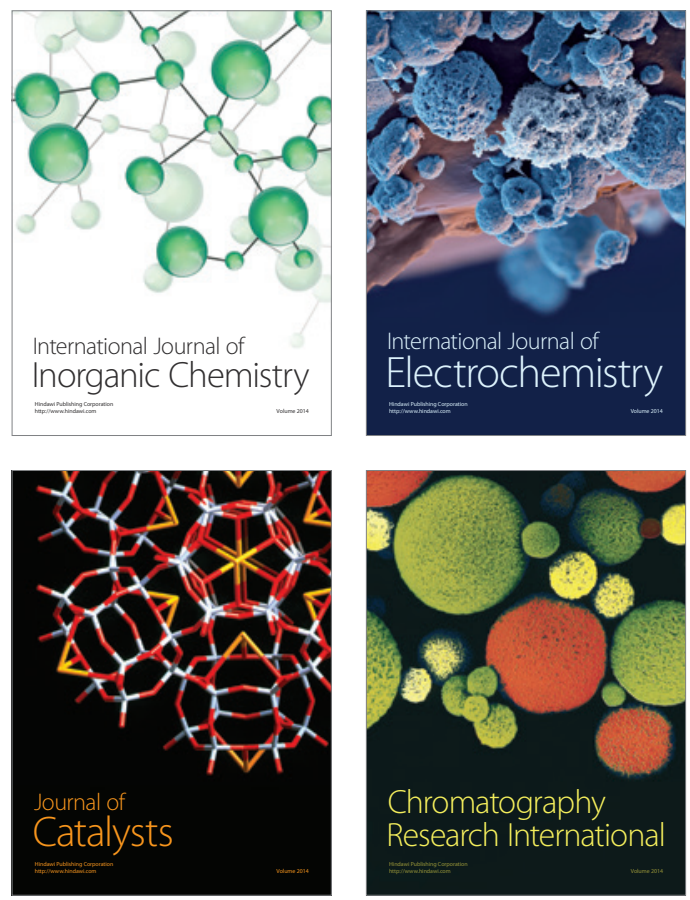
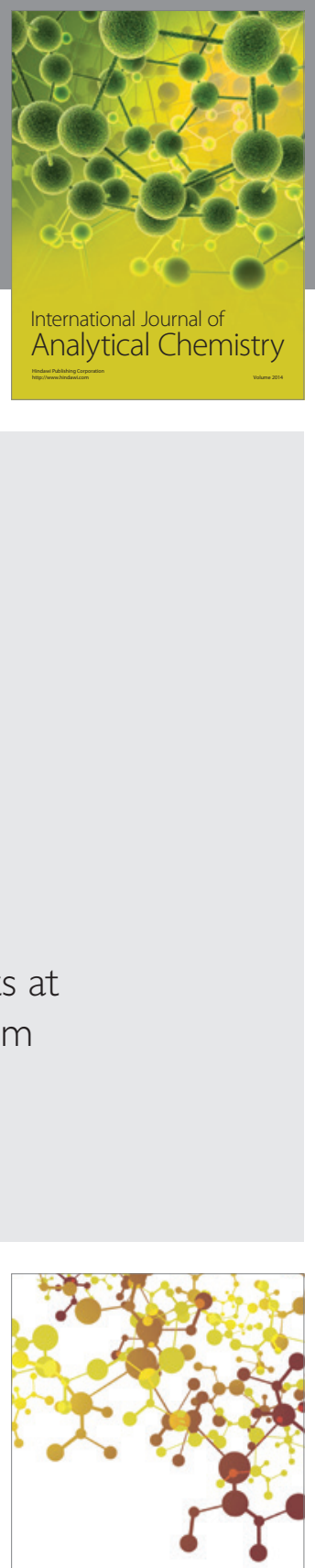

Journal of

Applied Chemistry
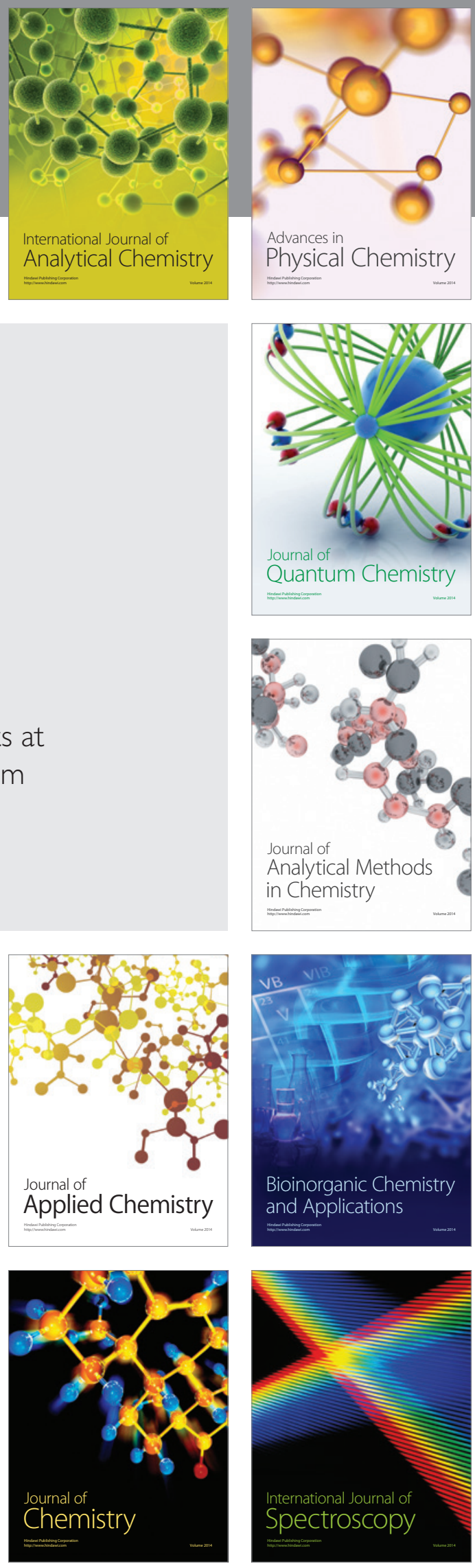